\title{
When supernovae explode in a starburst: transitions among AGN types
}

\author{
Itziar Aretxaga \\ Instituto Nacional de Astrofísica, Óptica y Electrónica, \\ Apartado Postal 51 y 216, Puebla, Pue., Mexico
}

\begin{abstract}
Among the Active Galactic Nuclei that have suffered type mutations, the classically classified Seyfert 2 NGC 7582 recently found at a Seyfert 1 stage, is perhaps one of the most puzzling cases, since it defies a well supported case for unification. We investigate the possibility that this type transition, common to another twelve AGN, is driven by supernovae exploding in a nuclear/circumnuclear starburst.
\end{abstract}

\section{Introduction}

Variability is a common characteristic of AGN. Most variability studies concentrate their attention on luminous Seyfert 1 nuclei and QSOs, which are known to develop prominent variations. But even among types which classically have been regarded as quiescent, LINERs and Seyfert 2 nuclei, there is an increasing number of reported cases of variations in both development of broad lines and increase of continuum luminosity.

\section{Seyfert 1 mutation of the classical Seyfert 2 NGC 7582}

The recent discovery that NGC 7582 has mutated into a type 1 Seyfert (Joguet et al. 1998; Aretxaga et al. 1999a) is perhaps one of the most puzzling cases. This classical Seyfert 2 shows phenomenology that has been interpreted in support of unified schemes of AGN (Antonucci 1993): a sharp-edged [OIII] outflow in the form of a cone is detected (Morris et al. 1985); optical spectropolarimetry does not reveal any hidden broad line region (BLR), but since the far-IR colours 60-25 $\mu \mathrm{m}$ are very red, the absence has been taken in support for an edge-on thick torus able to block even the light scattered towards the observer (Heisler et al. 1997); indeed a large column density of neutral $\mathrm{H}$ also blocks the hard $\mathrm{X}$-rays, implying a very large obscuration (Mulchaey et al. 1996). The presence of stars in the nucleus is now firmly established. There is a steep gradient of $\mathrm{H} \alpha$ perpendicular to the [OIII] cone, which reveals a $1 \mathrm{kpc}$ disk of $\mathrm{H}$ II regions oriented at $60^{\circ}$ from the plane of the galaxy (Morris et al. 1985). The CO absorption lines and large near-IR light-to-mass ratio are similar to those of $\mathrm{H}$ II galaxies and a factor of 5 larger than those of normal galaxies, indicating that red supergiants dominate the light of the inner $200 \mathrm{pc}$ at those wavelengths (Oliva et al. 1995). 


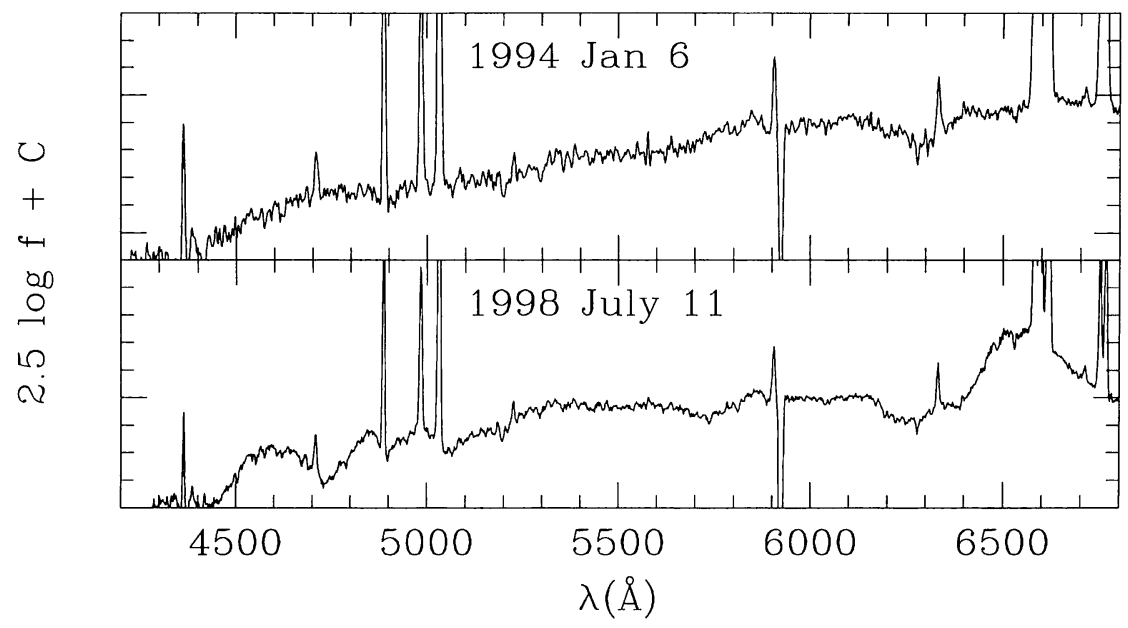

Figure 1. $\quad 2^{\prime \prime}$ extractions of spectra of the nucleus of NGC 7582.

Fig. 1 shows spectra of the nucleus of NGC 7582 as seen on January 6th 1994 (Cid Fernandes et al. 1998), the long known Seyfert 2 stage that lasted until 1998 June 20th (Halpern et al. 1998), and on July 11th, when it was discovered to have transited to a Seyfert 1 stage (Joguet et al. 1998).

This type transition, if rare, is not unprecedented. Three other classically classified Seyfert 2 nuclei and one LINER have suddenly developed broad lines in the past (Mrk 6, Mrk 993 and Mrk 1018 and NGC 1097), and seven Seyfert 1 and a radio-galaxy lost their broad components and became Seyfert 2 (NGC 1566, NGC 3516, NGC 4151, NGC 5548, NGC 6814, NGC 7603, Mrk 372 and 3C 390.3).

Some of these transitions have been interpreted in the framework of capture and disruption of stars by a supermassive black hole (Storchi-Bergmann et al. 1995) or by a variable reddening in the dusty torus that hides the BLR in Seyfert 2 (Goodrich 1989, 1995). In the case of NGC 7582 these two interpretations conflict with the high extinctions measured for the thick dusty torus ( $A_{V} \approx 230 \mathrm{mag}$ ), that would block all the optical light coming from the surroundings of the black hole (Aretxaga et al. 1999a). We can thus say, that the mutation experienced by NGC 7582 defies the framework postulated by unified schemes.

If the idea of a torus is to survive, the spectral change must be attributed to processes that occur around the torus, and which are not necessarily related to the central engine of the AGN. An attractive alternative is to attribute this Seyfert 1 activity to type IIn SN going off in the nuclear starburst detected at a radius $r<100 \mathrm{pc}$ (Oliva et al. 1995).

\section{Type IIn supernovae: SN $1988 \mathrm{Z}$}

The spectra of SN IIn are characterized by the presence of prominent narrow emission lines (hence the ' $n$ ') superposed on top of broad components of up 


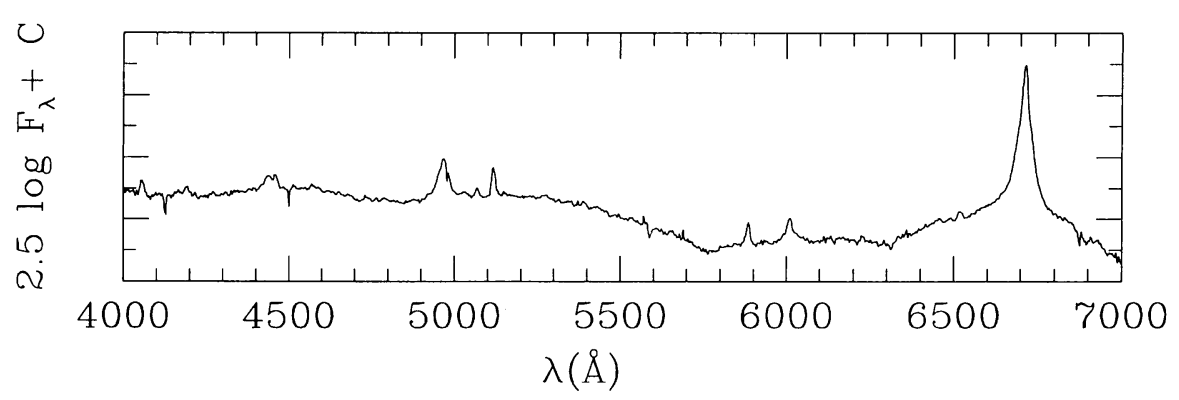

Figure 2. Spectrum of SN $1988 \mathrm{Z}$ at age $115 \mathrm{~d}$ from discovery.

to $\mathrm{FWHM} \approx 20,000 \mathrm{~km} \mathrm{~s}^{-1}$ which look very similar to those of Seyfert 1 nuclei and QSOs (Filippenko 1989), and don't show the characteristic broad P-Cygni signatures of standard SN. SN IIn are normally associated with regions of starformation.

Fig. 2 shows a spectrum of SN 1988Z, the best followed-up SN of this type, near maximum (Turatto et al. 1993). This is an exceptionally bright and peculiar SN in its spectro-photometric properties:

- It is characterized by an extremely slow decay of luminosity after maximum light, which makes it at day 600 approximately 5 mag brighter in $V$-band than standard type II SN (Stathakis \& Sadler 1991).

- It has a strong $\mathrm{H} \alpha$ emission, with peak luminosities of about $4 \times 10^{41} \mathrm{erg} \mathrm{s}^{-1}$ (for $\mathrm{H}_{0}=50 \mathrm{~km} \mathrm{~s}^{-1} \mathrm{Mpc}^{-1}$ ) at day 200 (Turatto et al. 1993). This prodigious luminosity is 5 orders of magnitude larger than that of SN 1987A.

- Very high-ionization coronal lines (e.g., [Fex] $76375 \AA$, [FexI] $77889-7892 \AA)$ are identified in the optical spectra, at least until day 492 (Turatto et al. 1993).

- At $2-20 \mathrm{~cm}$ it is one of the most powerful radio-SN in the sky, with peakluminosities up to 3000 times that of remnants like Cas A (Van Dyk et al. 1993).

- Even 6 yr after maximum the SN shows a hard X-ray emission (Fabian \& Terlevich 1996) of more than $10^{41} \mathrm{erg} \mathrm{s}^{-1}$.

- The total integrated energy radiated by this event in $8.5 \mathrm{yr}$ of evolution is at least $2 \times 10^{51} \mathrm{erg}$, and probably close to $10^{52} \mathrm{erg}$, in contrast with the canonical $10^{49} \mathrm{erg}$ of standard SN (Aretxaga et al. 1999b).

Fig. 3 shows a combined representation of the above properties. From this we derive that the output of energy is dominated by optical to X-ray emission, with a spectral energy distribution which strongly deviates from the classical black-body fit of typical SN (Aretxaga et al. 1999b).

These properties can be interpreted in the light of quick reprocessing of the kinetical energy released in the explosion by a dense circumstellar medium (CSM) (Terlevich et al. 1992; Aretxaga et al. 1999b), and thus explain the phenomenon as a young and compact supernova remnant (cSNR) rather than as a SN. Radiative cooling is expected to become important well before the thermalization of the ejecta is complete. As a result, the shocked material undergoes a rapid condensation behind both the leading and reverse shocks. These 


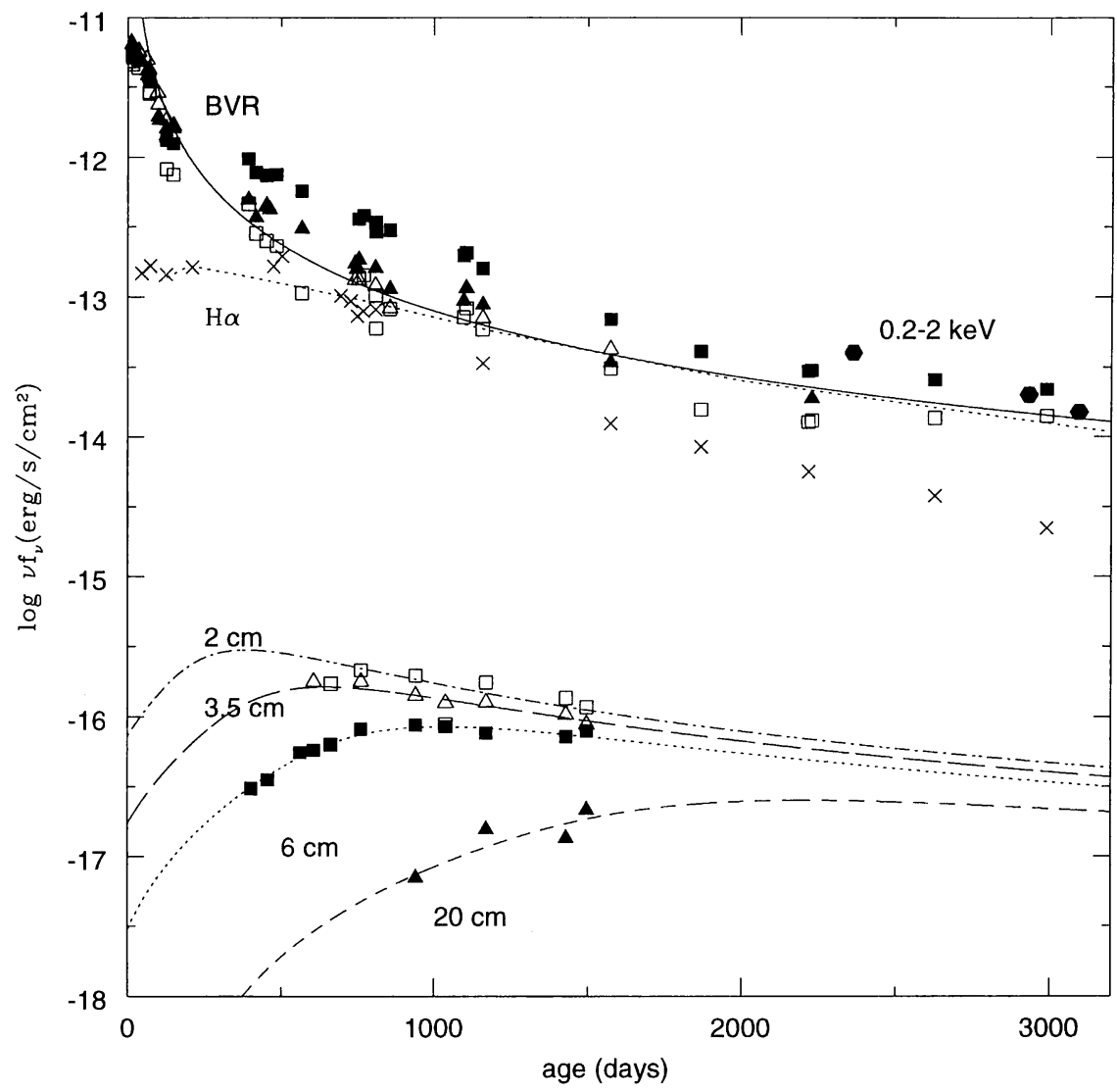

Figure 3. Evolution of the $\nu f_{\nu}$ light curve in radio to X-ray bands. In the upper part of the panel open triangles correspond to $B$-band, solid triangles to $V$-band, solid squares to $R$-band, empty squares to $R c$ band, solid hexagones to the ROSAT 0.2 to $2 \mathrm{keV}$ band and crosses to $\mathrm{H} \alpha$. The solid and dashed lines are models for the bolometric light and $\mathrm{H} \alpha$ evolution of a cSNR. In the lower part of the panel, VLA radio data at 2, 5.5, 6 and $20 \mathrm{~cm}$ is represented with empty squares, empty triangles, solid squares and empty triangles. The line fittings correspond to the models of Van Dyk et al. (1993).

high-density thin shells, the freely expanding ejecta and the still unperturbed interstellar gas are all ionized by the radiation produced in the shocks, and are responsible for the complex emission line structure observed in these objects.

In the case of SN 1988Z, a direct measurement of the CSM density was possible (Stathakis \& Sadler 1991). The value determined from the [OIII] $\lambda 4363 \AA$ to [OIII] $\lambda 5007 \AA$ forbidden line ratio in the early stages of the evolution is between $4-16 \times 10^{6} \mathrm{~cm}^{-3}$.

The semi-analytical behaviour of a cSNR (Terlevich et al. 1992) in comparison with the data of SN $1988 \mathrm{Z}$ is shown with lines in Fig. 3 and Fig. 6. 


\section{Starbursts that explode type IIn SNe}

There is little doubt that if a SN IIn goes off in the center of a normal galaxy, the nucleus would be classified as a Seyfert 1 while the prominent broad lines remain visible. In fact, there has been a succession of theoretical works that explain the phenomenology of lines and continuum at UV to near-IR wavelengths in Seyfert 1 nuclei in terms of a starburst that undergoes SN IIn explosions (see R.J. Terlevich, these Proceedings). From an age 10-60 Myr, a starburst sustains a SN rate directly proportional to the blue light emitted by the stars in the cluster, with a value that is almost independent on the initial mass function (Aretxaga \& Terlevich 1994a):

$$
\nu_{\mathrm{SN}} / L_{\mathrm{B}}^{\star} \approx 2 \times 10^{-11} \mathrm{yr}^{-1} \mathrm{~L}_{\mathrm{B}}^{\odot^{-1}} .
$$

If we take the light evolution of SN $1988 \mathrm{Z}$ as typical of the SN exploding in a cluster (but beware that SN IIn show some heterogeneity in their light curves, $c f$. Filippenko 1997), we can construct some models to predict type transitions generated by SN IIn (Aretxaga \& Terlevich 1994). Fig. 4 shows a Monte Carlo simulation of the light curve of a cluster of $\mathrm{M}_{B}^{\star} \approx-18.7$ mag (marked with a dashed line). The SN light evolution has been represented by the semi-analitical fit shown in Fig. 3, and each SN explosion is marked with an arrow.

According to the photo-ionization models for cSNR (Terlevich et al. 1992), the equivalent widths of $\mathrm{H} \alpha$ and $\mathrm{H} \beta$ change in time from $W_{\mathrm{H}_{\alpha}} / \nu_{\mathrm{SN}} \approx 37.7 \AA \mathrm{yr}$, $W_{\mathrm{H} \beta} / \nu_{\mathrm{SN}} \approx 6.1 \AA \mathrm{yr}$ for $4 t_{\mathrm{sg}}$ to $W_{\mathrm{H} \alpha} / \nu_{\mathrm{SN}} \approx 13.1 \AA \mathrm{yr}, W_{\mathrm{H} \beta} / \nu_{\mathrm{SN}} \approx 1.4 \AA \mathrm{yr}$ for $8 t_{\mathrm{sg}}$, where $t_{\mathrm{sg}}$ is the characteristic time of evolution of the cSNR. If we adopt $20 \AA$ as the observable limit below which an object is classified as a Seyfert 1.9 (in case $W_{\mathrm{H}_{\beta}} \lesssim 20 \AA$ but $W_{\mathrm{H}_{\alpha}} \gtrsim 20 \AA$ ) or a Seyfert 2 (in case $W_{\mathrm{H}_{\alpha}}, W_{\mathrm{H}_{\beta}} \lesssim 20 \AA$ ), the transitions take place when the total light emitted by the cluster (solid line in Fig. 4) is less than about 0.14 and 0.01 mag above the stellar level (dashed line) respectively. The activity level is recovered once the light curve crosses those limits in the opposite direction due to a new cSNR.

We can then estimate the time spent by these clusters as Seyfert nuclei of types 1.9 or 2 (Aretxaga \& Terlevich 1994b). The values obtained are just

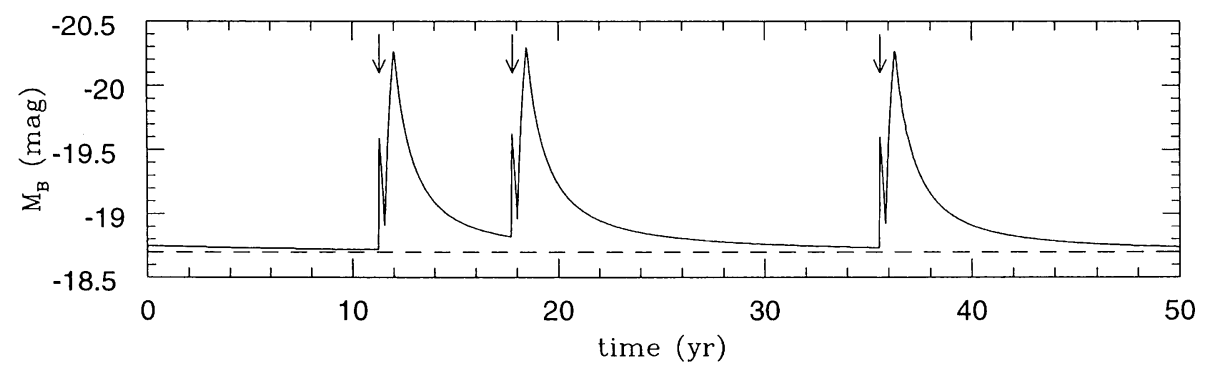

Figure 4. Theoretical light curve of a massive stellar cluster undergoing a $\mathrm{SN}$ rate $\nu_{\mathrm{SN}} \approx 0.1 \mathrm{yr}^{-1}$. The arrows in the diagram represent the moments in which the SN explodes and the dashed lines the luminosity level coming from main sequence stars (Aretxaga et al. 1997). 


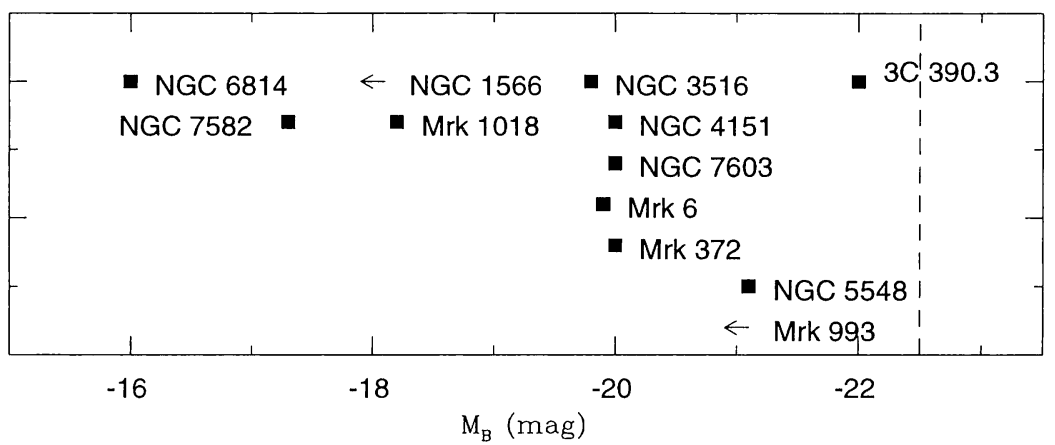

Figure 5. Number of transient AGN per luminosity interval. Squares represent mean luminosities, and arrows indicate upper limits to these quantities. The luminosity threshold for allowed transitions is marked with a line.

upper limits to the time spent in quiescent stages, since our approach ignores the secondary pulses that occur in the evolution of cSNR due to cooling instabilities. The less luminous systems are the ones that experience longer quiescent stages. In low-luminosity systems $\left(\mathrm{M}_{B}>-22.5 \mathrm{mag}\right)$ the low $\mathrm{SN}$ rates derived $\left(\nu_{\mathrm{SN}} \lesssim 1 \mathrm{yr}^{-1}\right)$ gives non negligible time scales for states in which no cSNR could contribute to the existence of broad lines in the spectrum, and thus transitions between Seyfert types 1 and 2 can be possible. Fig. 5 compares the luminosity of the transient AGN as compared with that theoretical limit, which lies above the measured luminosities.

\section{A type IIn SN in the nucleus of NGC 7582?}

The luminosity of the inner $3^{\prime \prime}$ of NGC 7582 before the transition is $V \approx 15$ mag. The luminosity profile at this stage is not very peaked, as shown in HST images, where the luminosity of the nucleus changes by 3 mag when using apertures ranging from 0.5 to 0.1 arcsec. The stellar populations of the nucleus at optical wavelengths are best represented by old ages $(t>100 \mathrm{Myr})$ contaminated in a $12 \%$ by a reddened $E_{B-V} \approx 0.6 \mathrm{mag}$ starburst (Schmitt et al. 1999 ). Therefore, the intrinsic luminosity of the starburst nucleus is about $\mathrm{M}_{B} \approx-17.3 \mathrm{mag}$ and the $\mathrm{SN}$ rate must then be $\nu_{\mathrm{SN}} \approx 0.02 \mathrm{yr}^{-1}$. If all the $\mathrm{SN}$ explosions generate SN IIn, there is a $33 \%$ probability of having detected a transition to a Seyfert 1 stage in the last $30 \mathrm{yr}$.

In order to investigate this possibility for the origin of the broad lines, profile and light evolution might be of interest. Fig. 6 plots the evolution of the $\mathrm{H} \alpha$ line width and luminosity of NGC 7582 as a function of time compared with the values of SN 1988Z (Aretxaga et al. 1999b) and the Seyfert 1 NGC 5548 (Korista et al. 1995). The data of NGC 7582 were obtained from various ESO telescopes (Aretxaga et al. 1999a), and from the $2.1 \mathrm{~m}$ in Cananea, Mexico (previously unpublished data). The spectra were internally flux-calibrated to the same relative scale using [NII] $\lambda 6583 \AA$ and, independently, also [OIII] $\lambda 5007 \AA$. The zeros in the time axis of Fig. 6 for the comparison are uncertain. We have opted to 

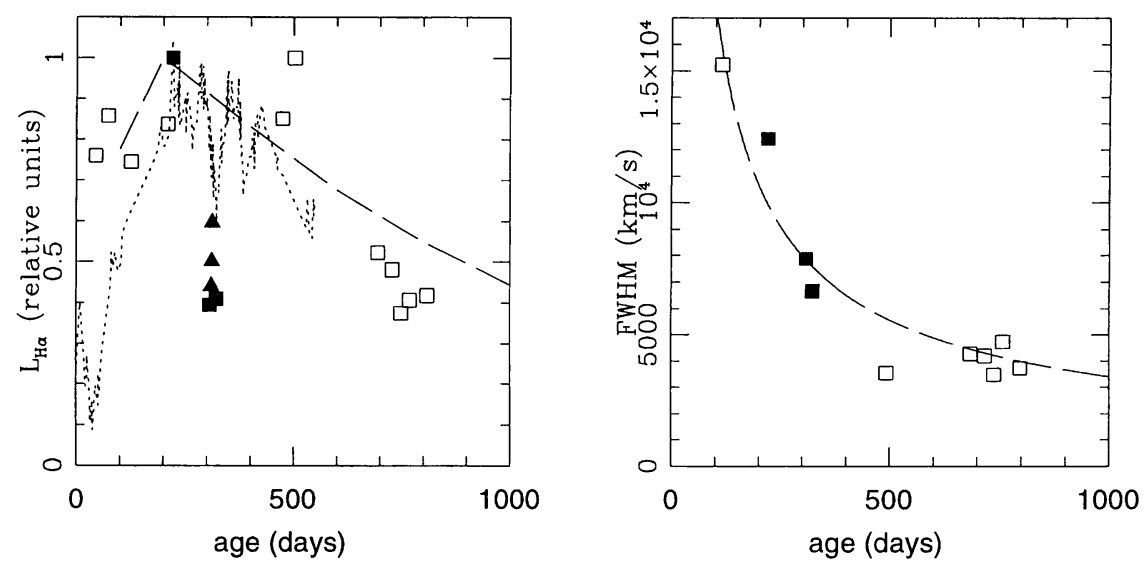

Figure 6. Evolution of $\mathrm{H} \alpha$ line width and luminosity for NGC 7582 (filled squares and triangles), SN $1988 \mathrm{Z}$ (empty squares), NGC 5548 (dotted line) and a cSNR model (dashed line).

match the light curves of SN 1988Z, NGC 7582 and NGC 5548 at the maximum in $\mathrm{H} \alpha$ light. This also matches the minimum in the light evolution of NGC 5548 as the onset of the 'elementary unit of variation' in Seyfert 1 nuclei (Cid Fernandes et al. 1997). All fluxes have been normalized to the maximum value. The line-width evolution of NGC 7582 closely resembles the early evolution of SN 1988Z, if the similarity of flux evolution is not clear due to the scarcity of the data. At these early stages, large deviations from the semi-analytical solution of the evolution of a cSNR, represented as a dashed line, are expected due to the formation and collision of the shell structures generated by the outer and reverse shocks as they sweep circumstellar and ejected material (Terlevich et al. 1992) . However, these light oscillations predicted by theory are as yet unchecked in real SN IIn. These oscillations are however clearly present in the behaviour of the light evolution of typical Seyfert 1 nuclei, as NGC 5548 represented with dotted lines. If the event seen in NGC 7582 is a cSNR in the nuclear starburst region, a behaviour similar to SN $1988 \mathrm{Z}$ and the peak of NGC 5548 represented in Fig. 6 is expected.

A close multi-wavelength monitoring of this object is thus required if we want to elucidate the mechanism that has created the broad-lines despite the supposedly still existing thick torus that blocks the inner nuclear region from the line of sight.

Acknowledgments. I would like to acknowledge my collaborators in the several projects that have been joined to shape this paper: S. Benetti, B. Joguet, D. Kunth, J. Melnick, A. Fabian, R.J. Terlevich and J.R. Valdes. Especial thanks go to B. Joguet and J.R. Valdes who were crucial in providing me with the data of NGC 7582, keeping the flow open until hours before my talk. 


\section{References}

Antonucci, R.R.J. 1993, ARAA 31, 473

Aretxaga, I., Terlevich, R.J. 1994a, MNRAS 269, 462

Aretxaga, I., Terlevich, R.J. 1994b, in: G. Tenorio-Tagle G. (ed.), Violent Star Formation: From 30 Doradus to QSOs, (Cambridge: CUP), p. 347

Aretxaga, I., Cid Fernandes, R., Terlevich, R.J. 1997, MNRAS 286, 271

Aretxaga, I., Joguet, B., Kunth, D. et al. 1999a, ApJL submitted

Aretxaga, I., Benetti, S., Terlevich, R.J., et al. 1999b, MNRAS in press

Cid Fernandes, R., Terlevich, R.J., Aretxaga I. 1997, MNRAS 289, 318

Cid Fernandes, R., Storchi-Bergmann, T., Schmitt, H.R. 1998, MNRAS 297, 579

Fabian, A.C., Terlevich, R.J. 1996, MNRAS 280, L5

Filippenko, A.V. 1989, ApJ 97, 726

Filippenko, A.V. 1997, ARAA 35, 309

Goodrich, R.W. 1989, ApJ 340, 190

Goodrich, R.W, 1995, ApJ 440, 141

Halpern, J.P., Kay, L.E., Leight, K.M. 1998, IAU Circ. 7027

Heisler, C.A., Lumsden, S.L., Bailey, J.A. 1997, Nature 385, 700

Joguet, B., Kunth, D., Terlevich, R.J. 1998, IAU Circ. 7024

Korista K., Alloin, D., Barr, P., Clavel, J., Cohen, R.D., et al. 1995, ApJS 97, 285

Morris, C.S., Ward, M., Whittle, M., et al. 1985, MNRAS 216, 193

Mulchaey, J.S., Wilson, A.S., Tsvetanov, Z. 1996, ApJ 467, 197

Oliva, E., Origlia, L., Kotilainen, J., Moorwood A. 1995, A\&A 301, 55

Schmitt, H.R., Storchi-Bergmann, T., Cid Fernandes, R. 1999, MNRAS 303, 173

Stathakis, R.A., Sadler, E.M. 1991, MNRAS 250, 786

Storchi-Bergmann, T., Eracleous, M., Livio, M., et al. 1995, ApJ 443, 617

Terlevich, R.J., Tenorio-Tagle, G., Franco, J. et al. 1992, MNRAS 255, 713

Turatto, M., Cappellaro, E., Danzinger, I.J., et al. 1993, MNRAS 262, 128

Van Dyk, S.D., Weiler, K.W., Sramek, R.A., Panagia, N. 1993, ApJ 419, L69

\section{Discussion}

Marchenko: The modeled (generated) light-curves remind me of the variations generated by a black-hole fed by passing stars. How typical could the variability you are describing be for the AGNs? How significant would be other channels, if any?

Aretxaga: As mentioned, variations among LINERS and Seyfert 2 galaxies are rare. There are other mechanisms proposed to explain the variations, the capture of stars being one of them. In the case of Seyfert 2 with obscuring tori, like NGC 7582, the problem for those models is that the torus itself wouldn't let the newly created BLR be observed in optical light, because the stripping of stellar material happens at a distance to the hole which is smaller than the tidal radius, i.e., around $10^{-5} \mathrm{pc}$. On the other hand, we know that nuclear starbursts are common in Seyfert 2 and, in particular, in NGC 7582 there is strong evidence of its existence. This starburst may even be surrounding the torus! It just takes one SN explosion to be a SN IIn to make that nucleus appear as a Seyfert 1.

Marston: (1) How much might you expect the placing of the slit of the spectroscope to affect your results? Do you place your slit in exactly the same place each time? 
(2) If this is not a particular problem, then is there a correlation between varying spectrum and variability in extinction?

Aretxaga: (1) We placed the slit at the brightest nuclear spot. We have done the astrometry of the nucleus before and after the transition and we find that the centroid of the nucleus remains in the same position, inside an error box of 0 "' 16 .

(2) The continuum variability between the Seyfert 2 and the Seyfert 1 stage on July 11th 1998 is inconsistent with a pure variation in extinction, at least if the extinction law is taken to be the local one (see Aretxaga et al. 1999 for details).

Polcaro: In 1981, more or less at the time of the optical minimum, we took hard X-ray (20-80 keV) spectra of NGC 4151, and found its spectrum quite different from that we took in the previous year. We found also a similar effect, in other experiment, in MCG 8-11-11. How does your model fits this experimental evidence?

Aretxaga: At minimum light, when no cSNR contributes to the existence of broad lines, the spectral energy-distribution of the AGN is expected to be different from that at minimum light. SN IIn like SN 1988Z, for instance, have most of their energy output at optical to X-ray frequencies, and thus the optical transition is probably accompanied by an X-ray transition too.

Langer: Your evidence for the AGN-SN scenario is quite nice. However, I am puzzled by one thing. The observed Type IIn SNe are found, as I understand, in disks and spiral arms. Would one imagine at one particular location in the disk of a spiral galaxy a $\mathrm{SN}-$ rate of $1 \mathrm{SN} / \mathrm{yr}$, this location would certainly quickly be evacuated. In order for this not to occur in the AGN nucleus, the conditions there (density, pressure) must thus be different. Does this not imply that a comparison of SN IIn spectra and Seyfert 1 spectra may be meaningless?

Aretxaga: The radius of material swept by one of these SNIIn is actually not very large due to the high density of circumstellar material. In the case of SN 1988Z this is about $0.02 \mathrm{pc}$ after 8.8 years. But in nuclei with high SN-rates, the superposition of SN bubbles could actually break the disks into mildly collimated outflows.

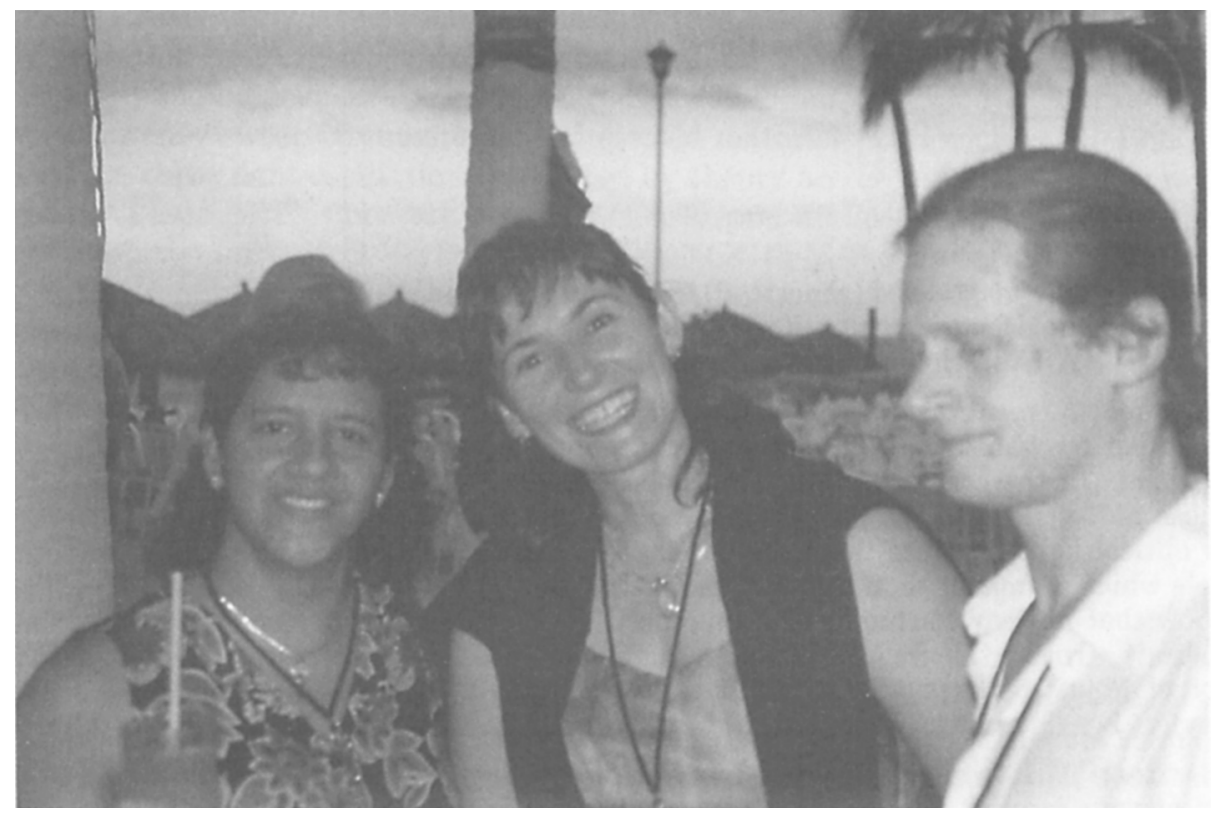

